\title{
A Treecode for Accurate Force Calculations*
}

\author{
Kasthuri Srinivasan ${ }^{\star \star}$ and Vivek Sarin \\ Department of Computer Science, Texas A\&M University, \\ College Station, TX, USA \\ \{kasthuri, sarin\}@cs.tamu.edu
}

\begin{abstract}
A novel algorithm for computing forces in N-body simulations is presented. This algorithm constructs a hierarchical tree data structure and uses multipole expansions for the inverse square law. It can be considered as a multipole-based variant of Barnes-Hut algorithm [2]. The key idea here is the representation of forces (gravitational or electrostatic) through a multipole series by using the relationship between ultraspherical and Legendre polynomials. The primary advantage of this algorithm is the accuracy it offers along with the ease of coding. This method can be used in the simulation of star clusters in astrophysical applications in which higher order moments are expensive and difficult to construct. This method can also be used in molecular dynamics simulations as an alternative to particle-mesh and Ewald Summation methods which suffer from large errors due to various differentiation schemes. Our algorithm has $O\left(p^{3} N \log N\right)$ complexity where $p$ is typically a small constant.
\end{abstract}

\section{Introduction}

N-body simulations have become very important in theoretical and experimental analysis of complex physical systems. A typical N-body problem models physical domains where there is a system of $N$ particles (or bodies), each of which is influenced by gravitational forces or electrostatic forces of all other particles. The naive brute force algorithm to compute the forces acting on each particle based on particle-particle interaction is an $O\left(N^{2}\right)$ algorithm. There are various approximation algorithms with lower complexity that have been proposed for these simulations. In the simulation of gravitational forces in astrophysics, BarnesHut algorithm [2] and its variants are used. For electrostatic force simulations occurring in molecular dynamics (MD), Fast Multipole algorithm (FMM) [5], particle-mesh methods like P3M [8] and Ewald Summation techniques [7] are widely applied.

For two particles of strengths $q_{1}$ and $q_{2}$, the force acting on $q_{1}$ due to $q_{2}$, is given by the inverse square law,

$$
\boldsymbol{F}=\frac{q_{1} q_{2}}{\left|\boldsymbol{r}_{\mathbf{1 2}}\right|^{2}} \cdot \frac{\boldsymbol{r}_{\mathbf{1 2}}}{\left|\boldsymbol{r}_{\mathbf{1 2}}\right|},
$$

\footnotetext{
* This work has been supported in part by NSF under the grants NSF-CCR0113668 and NSF-CCR0431068.

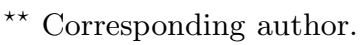


where $\left|\boldsymbol{r}_{\mathbf{1 2}}\right|$ is the distance between the particles. In this paper, the constant of proportionality (like the gravitational constant) is assumed to be 1. Even though the above law is applicable to both gravitational and electrostatic interactions, the required accuracy determines the algorithms to be used. The Barnes-Hut algorithm is an easy-to-code algorithm used in most astrophysical simulations where lower accuracy is often preferred (around 1\%) over reduced execution time. In astrophysical simulations, the force is approximated by a multipole series expansion with respect to the center of mass. The first moments are large enough (since the masses are all positive) to approximate the forces. Therefore, the monopole moment and the first two moments in the multipole series provide enough accuracy. On the other hand, in MD simulations, higher accuracy is needed because the distance and time scales involved are very different from astrophysical problems. The Barnes-Hut scheme can be adapted to MD simulations by adding more terms to the multipole series. For electrostatic force simulations, however, the distribution of positive and negative charges impedes the numerical robustness of the Barnes-Hut algorithm [6]. Methods like Ewald Summation, particle-mesh based approaches like P3M, and FMM are preferred over Barnes-Hut algorithm for electrostatic problems.

In contrast, in the investigation of collisional astrophysical systems, namely star clusters and galactic nuclei, there is a need for high accuracy. Such applications require $|\delta E / E| \ll 0.04 N^{-1} t / t_{c r}$ where $t$ and $t_{c r}$ are time parameters, $|\delta E / E|$ is the relative energy accuracy and $N$ is the number of stars in the system [4]. For a system of $10^{3}$ stars, this means an upper bound of $10^{-5}$ on the error. In order to achieve such high accuracy, McMillan and Aarseth [3] use octupole terms in the multipole expansion which are quite cumbersome to construct. Moreover, increased accuracy is needed for larger systems in order to limit the cumulative errors on core-collapse time scales to acceptable levels. To our knowledge there is no prior work that uses higher order moments than octupoles.

In the case of electrostatic force interactions, particle-mesh algorithms like P3M and Ewald Summation techniques compete with FMM [9, 10]. Despite the superior asymptotic scaling of FMM when compared to earlier methods $[O(N)$ versus $O(N \log N)$ ], FMM has some disadvantages. Apart from the obvious difficulty in coding, FMM suffers from lack of energy conservation unless very high accuracy is employed [11. In addition, special considerations regarding the degree of force interpolation must be taken into account to conserve momentum. Pollock and Glosi [10] discuss other advantages of P3M over FMM. Even in force interpolation methods used in particle-mesh algorithms and Ewald Summation methods, a general statement of accuracy seems to be difficult. The error analysis of force calculations in these methods are quite involved and they provide a fair comparison only if done at the same level of accuracy. The discretization methods introduce new sources of errors in addition to the ones originating from real and reciprocal space cutoffs. Furthermore, investigation of errors during force evaluation depends on several parameters such as mesh-size, interpolation orders and 
differentiation schemes. Deserno and Holm [12] illustrated remarkable differences in accuracy for these methods.

In this paper, we present a novel algorithm for accurate force calculations in N-body problems. This algorithm combines the ease of the Barnes-Hut method with a very high accuracy. Instead of using higher order moments, the algorithm uses additional terms of a multipole series to increase the accuracy. Our method can be used effectively in the calculation of star cluster interactions in which accuracy has been limited by octupole moments. Unlike particle-mesh and Ewald Summation based methods, our algorithm does not involve differentiation (either differentiation in Fourier space, analytic differentiation or discrete differentiation) to calculate the forces. Forces are directly computed through the series. This is especially useful where force interpolation is affected by conservation laws. Further, it may be of interest to note that the complexity of this algorithm is $O\left(p^{3} N \log N\right)$, which is comparable to that of particle-mesh algorithms that use Fast Fourier Transforms.

The paper is organized as follows: Section 2 discusses the multipole expansions for forces calculations. Section 3 describes the algorithm, analyzes its complexity and discusses implementation issues. Section 4 presents numerical experiments. Conclusions are presented in Section 5.

\section{Multipole Expansion for Forces}

A multipole based tree code for computing potentials of the form $r^{-\lambda}, \lambda \geq 1$, was introduced in 1]. In this paper, we extend the result to evaluate the forces. The reader is advised to refer [1] for some of the discussions in this section. The key idea in computing the potentials of the form $r^{-\lambda}$ is the use of ultraspherical polynomials in a manner analogous to the use of Legendre polynomials for the expansion of the Coulomb potential $r^{-1}$. Ultraspherical polynomials (or Gegenbauer polynomials) are generalizations of the Legendre polynomials in terms of the underlying generating function. That is, if $x, y \in \Re$, then

$$
\frac{1}{\left(1-2 x y+y^{2}\right)^{\lambda / 2}}=\sum_{n=0}^{\infty} C_{n}^{\lambda}(x) y^{n},
$$

where $C_{n}^{\lambda}(x)$ is an ultraspherical polynomial of degree $n$. They are also higher dimensional generalizations of Legendre polynomials [14, 15] in the sense that ultraspherical polynomials are eigenfunctions of the generalized angular momentum operator just as Legendre polynomials are eigenfunctions of the angular momentum operator in three dimensions. One can refer to 14 for a list of similarities between them. The above generating function can be used to expand $r^{-\lambda}$. In [1] we derived the following multipole expansion theorem for $r^{-\lambda}$ potentials.

Theorem 1 (Multipole Expansion for Potentials) [1]. Suppose that $k$ charges of strengths $\left\{q_{i}, i=1, \ldots k\right\}$ are located at the points $\left\{Q_{i}=\left(\rho_{i}, \alpha_{i}, \beta_{i}\right), i=\right.$ $1, \ldots, k\}$, with $\left|\rho_{i}\right|<a$. Then, for any point $P=(r, \theta, \phi)$ with $r>a$, the potential $\Phi(P)$ is given by 


$$
\Phi(P)=\sum_{n=0}^{\infty} \sum_{m=0}^{\lfloor n / 2\rfloor} \frac{1}{r^{n+\lambda}} \mathbf{M}_{\mathbf{n}}^{\mathbf{m}} \cdot \mathbf{Y}_{\mathbf{n}, \mathbf{m}}(\theta, \phi)
$$

where

$$
\begin{gathered}
\mathbf{M}_{\mathbf{n}}^{\mathbf{m}}=\sum_{i=1}^{k} q_{i} \rho_{i}^{n} B_{n, m}^{\lambda} \overline{\mathbf{Y}_{\mathbf{n}, \mathbf{m}}}\left(\alpha_{i}, \beta_{i}\right), \\
\mathbf{Y}_{\mathbf{n}, \mathbf{m}}^{\mathbf{T}}(x, y)=\left[Y_{n-2 m}^{-(n-2 m)}(x, y), Y_{n-2 m}^{-(n-2 m)+1}(x, y), \ldots, Y_{n-2 m}^{(n-2 m)}(x, y)\right]
\end{gathered}
$$

is a vector of spherical harmonics of degree $n-2 m$ and

$$
B_{n, s}^{\lambda}=\frac{(\lambda)_{n-s}(\lambda-1 / 2)_{s}}{(3 / 2)_{n-s} s !}(2 n-4 s+1) .
$$

Furthermore, for any $p \geq 1$,

$$
\left|\Phi(P)-\sum_{n=0}^{p} \sum_{m=0}^{\lfloor n / 2\rfloor} \frac{\mathbf{M}_{\mathbf{n}}^{\mathbf{m}}}{r^{n+\lambda}} \cdot \mathbf{Y}_{\mathbf{n}, \mathbf{m}}(\theta, \phi)\right| \leq \frac{A B}{r^{\lambda-1}(r-a)}\left(\frac{a}{r}\right)^{p+1},
$$

where $A=\sum_{i=1}^{k}\left|q_{i}\right|$ and $B=\sum_{m=0}^{\lfloor n / 2\rfloor}\left|B_{n, m}^{\lambda}\right|$.

The multipole expansion for force computations can now be deduced as a corollary to the above theorem.

Corollary 1 (Multipole Expansion for Forces). Suppose that $k$ particles of strengths $\left\{q_{i}, i=1, \ldots k\right\}$ are located at points whose position vectors are $\left\{\boldsymbol{\rho}_{i}, i=1, \ldots, k\right\}$ and let $\left\{Q_{i}=\left(\left|\boldsymbol{\rho}_{i}\right|, \alpha_{i}, \beta_{i}\right), i=1, \ldots, k\right\}$ denote their spherical coordinates with $\left|\boldsymbol{\rho}_{i}\right|<a$. Then, for any vector $\boldsymbol{r}$ with coordinates $P=(|\boldsymbol{r}|, \theta, \phi)$ and $|\boldsymbol{r}|>$ a, the $c^{\text {th }}$ component of the force, $F_{c}(P)$, is given by

$$
F_{c}(P)=\sum_{n=0}^{\infty} \sum_{m=0}^{\lfloor n / 2\rfloor} \frac{1}{|\boldsymbol{r}|^{n+3}}\left[\left(\boldsymbol{r}_{c}\right) \mathbf{M}_{\mathbf{n}}^{\mathbf{m}}-{ }_{c} \mathbf{V}_{\mathbf{n}}^{\mathbf{m}}\right] \cdot \mathbf{Y}_{\mathbf{n}, \mathbf{m}}(\theta, \phi)
$$

where

$\mathbf{M}_{\mathbf{n}}^{\mathbf{m}}=\sum_{i=1}^{k} q_{i}\left|\boldsymbol{\rho}_{\boldsymbol{i}}\right|^{n} B_{n, m}^{3} \overline{\mathbf{Y}_{\mathbf{n}, \mathbf{m}}}\left(\alpha_{i}, \beta_{i}\right), \quad{ }_{c} \mathbf{V}_{\mathbf{n}}^{\mathbf{m}}=\sum_{i=1}^{k} \boldsymbol{\rho}_{c i}\left(q_{i}\left|\boldsymbol{\rho}_{\boldsymbol{i}}\right|^{n} B_{n, m}^{3} \overline{\mathbf{Y}_{\mathbf{n}, \mathbf{m}}}\left(\alpha_{i}, \beta_{i}\right)\right)$.

Here $\boldsymbol{r}_{c}$ and $\boldsymbol{\rho}_{c i}$ are the $c^{\text {th }}$ component of $\boldsymbol{r}$ and $\boldsymbol{\rho}_{i}$, respectively. Furthermore, for any $p \geq 1$, the approximation $\hat{F}_{c}^{p}(P)$ obtained by truncating the expression in ([6) after $p$ terms, satisfies

$$
\left\|F_{c}(P)-\hat{F}_{c}^{p}(P)\right\|_{2} \leq \frac{A B}{|\boldsymbol{r}|^{2}(|\boldsymbol{r}|-a)}\left(\frac{a}{|\boldsymbol{r}|}\right)^{p+1}(|\boldsymbol{r}|+a),
$$

where $A=\sum_{i=1}^{k}\left|q_{i}\right|$ and $B=\sum_{m=0}^{\lfloor n / 2\rfloor}\left|B_{n, m}^{\lambda}\right|$. 
Proof. We note that $\boldsymbol{r}_{\mathbf{1 2}}=\boldsymbol{r}-\boldsymbol{\rho}_{1}$, and therefore, (11) can be written as

$$
\boldsymbol{F}(P)=\frac{q_{1}}{\left|\boldsymbol{r}_{\mathbf{1 2}}\right|^{3}} \boldsymbol{r}-\frac{q_{1}}{\left|\boldsymbol{r}_{\mathbf{1 2}}\right|^{3}} \boldsymbol{\rho}_{1} .
$$

Using $\lambda=3$ in (3) for $1 /\left|\boldsymbol{r}_{\mathbf{1 2}}\right|^{3}$, the proof follows through the superposition of particles at $\left\{\boldsymbol{\rho}_{i}, i=1, \ldots, k\right\}$. The error bound can be obtained from (5) by using the triangle inequality $\left|\boldsymbol{r}_{\mathbf{1 2}}\right| \leq|\boldsymbol{r}|+\left|\boldsymbol{\rho}_{1}\right| \leq|\boldsymbol{r}|+a$.

\section{Treecode for Force Calculations}

The treecode to compute the forces can be viewed as a variant of Barnes-Hut scheme that uses only particle-cluster multipole evaluations. The method works in two phases: the tree construction phase and the force computation phase. In the tree construction phase, a spatial tree representation of the domain is computed. At each step of this phase, if the domain contains more than one particle, it is recursively divided into eight equal sub-domains. This process continues until each sub-domain has at most one particle.

Each internal node in the tree computes and stores multipole series representation of the particles within its sub-domain. Note that we don't have the means to compute translations of multipole coefficients from child to parent nodes. We must compute the multipole coefficients at every internal node directly from the particles contained in the node. These coefficients are obtained using Corollary 1. Once the tree has been constructed, force at a point is computed using the multipole coefficients of a subset of the nodes in the tree, which are sufficiently far away from the point. A specific constant $\alpha$ is used to check if a node is far enough from the evaluation point. Given the distance of a point from the center of the sub-domain, $d$, and the side of the box, $r$, a point is considered to be far away from a node if the multipole acceptance criterion $(M A C)$ defined as, $d / r$, is greater than $\alpha$. For each particle, the algorithm proceeds by applying $M A C$ to the root of the tree to determine whether an interaction can be computed; if not, the node is expanded and the process is repeated for each of its eight children. If the node happens to be a leaf, then the force is calculated directly by using the particles in the node. In order to improve the computational efficiency of the algorithm, the minimum number of particles in any leaf box can be set to a constant $s$. The pseudo-code for this method can be found in [1].

It can be seen from Corollary 1 that the complexity of computing the multipole coefficients at each level of the tree is $O\left(p^{3} N\right)$, where $N$ is the number of particles in the system and $p$ is the multipole degree. Since there are $\log N$ levels in the tree for a uniform particle distribution, the total cost of computing the multipole coefficients is $O\left(p^{3} N \log N\right)$. Similarly, it can be seen that the complexity of the force evaluation phase is $O\left(p^{3} N \log N\right)$. Thus, the overall complexity for the algorithm is $O\left(p^{3} N \log N\right)$.

The algorithm has been implemented in $\mathrm{C}++$ programming language. The code first constructs the oct-tree and the multipole coefficients are calculated 
along with the tree construction. Standard Template Library (STL) data structures were used for the dynamically adaptive tree construction phase. The spherical symmetry of $r^{-3}$ potentials, when expressed in spherical coordinates, leads to efficient computations. For example, spherical harmonics for $r^{-1}$ potential is computed instead of computing the vector of spherical harmonics given in Theorem 11. Thus, computing $O\left(p^{2}\right)$ spherical harmonics for each particle is enough to compute $O\left(p^{3}\right)$ multipole coefficients, which results in significant time reduction for large systems. The constants defined in (4) are also precomputed and used in the force evaluation phase to reduce the overall computation time.

\section{Experiments}

In this section, we present results of numerical experiments performed on a 2.4 GHz, 512 MB Intel P4 PC running Red Hat 9.0. Due to space constraints we discuss two experiments only. In these tests, a direct summation code is the benchmark for comparing errors and execution time. For star-cluster applications, Mcmillan and Aarseth 3] compare accuracies for a problem with $N=10^{3}$. They target median force error below $10^{-4}$ and achieve it using octupole moments for $M A C<0.5$. Fig 1 shows the error in the force $F$ incurred by truncating expression (6) after $p$ terms. The relative error $\delta F / F$ is defined by

$$
\left(\frac{\delta F}{F}\right)^{2}=\frac{1}{N} \sum_{i=1}^{N}\left(\frac{\left|F_{\text {tree }}-F_{\text {direct }}\right|}{F_{\text {scale }}}\right)^{2},
$$

where $F_{\text {direct }}$ is the force determined by direct summation, $F_{\text {tree }}$ is the value returned by the tree algorithm and $F_{\text {scale }}=\sum_{i j} m_{i} /\left(r_{i j}^{\prime}\right)^{2}$ is a characteristic scale against which accuracy is measured. Here $m_{i}$ 's are the masses and the sums are taken over all particles in the system. Fig 1)(a) illustrates the effect of $M A C$ on error for a uniform distribution of $10^{3}$ particles with random masses in the range $[0,1]$. The maximum number of particles in leaf boxes were fixed at 10. For $M A C=0.9$ we see that an accuracy of $10^{-5}$ is reached using multipole degree 2. Also, as $M A C$ decreases, the error reduces proportionally. For a typical $M A C$ between 0.6 and 0.7 used in the Barnes-Hut algorithm, we have an order of magnitude reduction in the error by increasing the multipole degree. In [3], for $M A C<0.3$ the accuracy of the octupole component of the force deteriorates markedly. In our case, we see that dramatic reduction in the error is obtained as we increase the multipole degree. Also, Mcmillan et al. require $M A C<0.5$ for accurate computations. Since the amount of direct computation is inversely proportional to $M A C$, it may be expensive to select such small $M A C$ for large systems.

Fig 1(b) and Fig 2] demonstrate the effect of $M A C$ on accuracy and execution time for $10^{4}$ particles in random uniform distribution. Each particle carries a random positive or a negative charge. This test is carried out to verify the numerical robustness and consistency of our approach for electrostatic force interactions. As before, the multipole degree was allowed to vary between 2 and 4, and the number 


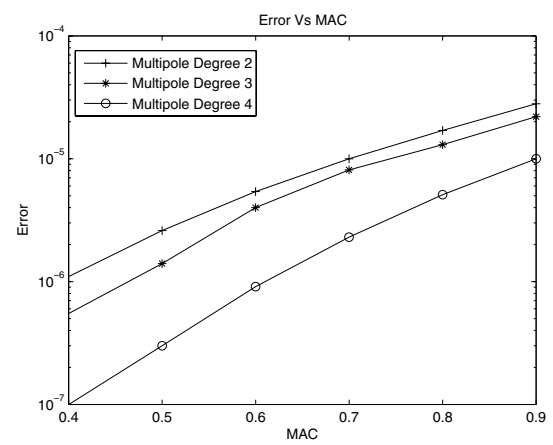

(a)

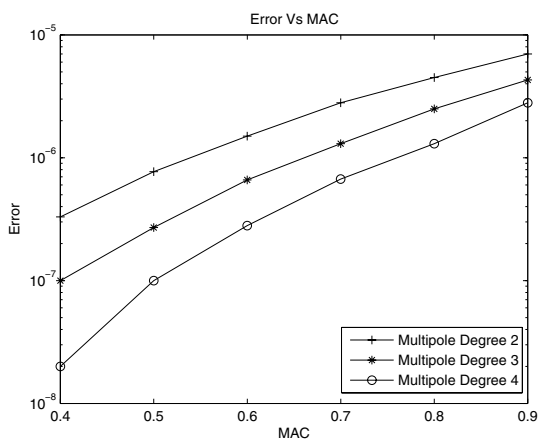

(b)

Fig. 1. Error Vs MAC with multipole degrees $p=2,3$ and 4 for (a) 1000 particles with positive masses (b) 10000 particles with positive and negative charges

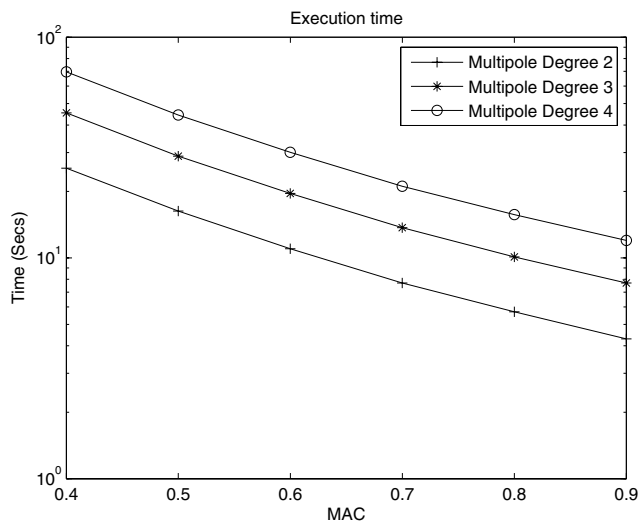

Fig. 2. Execution time for 10000 particles of positive and negative strengths

of particles per leaf box was fixed at 10. In Fig 2, we see a rapid increase in the execution time as we increase the multipole degree whereas the growth is smaller with respect to the MAC. In order to improve the accuracy for a small system, it is better to reduce the MAC than increase the multipole degree. However, treebased force evaluation schemes are quite inefficient for small number of particles because the overhead involved in manipulating and traversing the tree data structure is fairly large. For $N>10^{4}$, the treecode presented in this paper is expected to outperform the direct summation algorithm in execution time.

\section{Conclusion}

This paper presents an efficient algorithm for accurate calculation of forces using spherical coordinates. The proposed algorithm is superior to existing methods in which accuracy is limited by the choice of octupole moments and differentiation 
schemes. Our algorithm combines the ease of the Barnes-Hut algorithm with accurate force calculation, and can be used to compute gravitational forces in astrophysical simulations and electrostatic forces in molecular dynamics.

\section{References}

1. K. Kasthuri Srinivasan, H. Mahawar and V. Sarin. A Multipole Based Treecode Using Spherical Harmonics for the Potentials of the Form $r^{-\lambda}$. Proceedings of the International Conference on Computational Science (ICCS), Lecture Notes in Computer Science, Springer-Verlag, Vol. 3514, pp. 107-114, Atlanta, GA, May 2005.

2. J. Barnes and P. Hut. A Hierarchical $\mathrm{O}(n \log n)$ Force Calculation Algorithm. Nature, Vol. 324:446-449, (1986).

3. S.L.W. Mcmillan and S.J. Aarseth. An $O(N \log N)$ Integration Scheme for Collisional Stellar Systems. The Astrophysical Journal, Vol. 414, 200-212 (1993).

4. S. Pfalzner and P. Gibbon. Many-Body Tree Methods in Physics. The Cambridge University Press, Cambridge, Massachusetts (1996).

5. L. Greengard. The Rapid Evaluation of Potential Fields in Particle Systems. The MIT Press, Cambridge, Massachusetts (1988).

6. J. Board and K. Schulten. The Fast Multipole Algorithm. IEEE, Computing in Science and Engineering, Vol. 2, No. 1, January/February (2000).

7. P. Ewald. Die Berechnung Optischer und Elektrostatischer Gitterpotentiale. Ann. Phys., Vol.64, 253-287 (1921).

8. R.W. Hockney J.W. Eastwood. Computer Simulation Using Particles, New York: McGraw-Hill (1981)

9. C. Sagui and T. Darden. Molecular Dynamics Simulation of Biomolecules: LongRange Electrostatic Effects . Annu. Rev. Biophys. Biomol. Struct Vol. 28, 155-179 (1999).

10. E. Pollock and J. Glosli. Comments on PPPM, FMM and Ewald Method for Large Periodic Coulombic Syatems. In Comp. Phys. Comm., Vol. 95, 93-110 (1996).

11. T. Bishop, R. Skeel and K. Schulten. Difficulties with Multiple Time Stepping and Fast Multipole Algorithm in Molecular Dynamics. J. Comp. Chem., Vol. 18 1785-1791 (1997).

12. M. Deserno and C. Holm. How to Mesh up Ewald Sums: A Theoretical and Numerical Comparison of Various Particle Mesh Routines . J.Comp.Phys, Vol. 109 7678-7692 (1998).

13. E.J. Weniger. Addition Theorems as Three-Dimensional Taylor Expansions. International Journal of Quantum Chemistry, Vol.76, 280-295 (2000).

14. John Avery. Hyperspherical Harmonics, Kluwer Academic Publishers (1989)

15. Claus Müller. Analysis of Spherical Symmetries in Euclidean Spaces, SpringerVerlag (1991) 